\title{
Ultrafast and Highly Sensitive Chemically Functionalized Graphene Ox- ide Based Humidity Sensors: Harnessing the Device Performances via the Supramolecular Approach
}

\author{
Cosimo Anichini ${ }^{1}$, Alessandro Aliprandi ${ }^{1}$, Sai Manoj Gali², Fabiola Liscio ${ }^{3}$, Vittorio Morandi ${ }^{3}$, \\ Andrea Minoia ${ }^{2}$, David Beljonne ${ }^{2,}$, Artur Ciesielski1, ${ }^{*}$ and Paolo Samorì ${ }^{1,}$ \\ ${ }^{1}$ Université de Strasbourg, CNRS, ISIS, 8 alleé Gaspard Monge, 67000 Strasbourg, France. \\ 2 CMN, Université de Mons, Place du Parc 20, 7000 Mons, Belgium. \\ ${ }^{3}$ Istituto per la Microelettronica e Microsistemi (IMM) - CNR, via Gobetti 101, 40129 Bologna, Italy.
}

KEYWORDS: humidity sensing • graphene oxide $\bullet$ chemical functionalization $\bullet$ supramolecular chemistry $\bullet$ chemical sensing

ABSTRACT: Humidity sensors are gaining increasing attention because of their relevance for well-being. To meet the ever-
growing demand for new cost-efficient materials with superior performances, graphene oxide (GO) based relative humidity
sensors have emerged recently as low-cost and highly sensitive devices. However, current GO-based sensors suffer from
important drawbacks including slow response and recovery, as well as poor stability. Interestingly, reduced GO (rGO) ex-
hibits higher stability, yet accompanied by a lower sensitivity to humidity due to its hydrophobic nature. With the aim of
improving the sensing performances of rGO, here we report on a novel generation of humidity sensors based on a simple
chemical modification of rGO with hydrophilic moieties, i.e. tetraethylene glycol chains. Such hybrid material exhibits an
outstandingly improved sensing performance compared to pristine rGO such as high sensitivity (31 \% increase in electrical
resistance when humidity is shifted from 2 to $97 \%)$, an ultrafast response (25 ms) and recovery in the sub-second time-
scale, low hysteresis (1.1\%), excellent repeatability and stability as well as high selectivity towards moisture. Such highest
key performance indicators demonstrate the full potential of 2D materials when decorated suitably designed supramolecu-
lar receptors to develop the next generation of chemical sensors of any analyte of interest.

\section{INTRODUCTION}

Sensors capable of measuring the relative humidity with a high precision are key devices for monitoring the environment and play an important role in our daily life, industry, agriculture, bio-medical and environmental fields. ${ }^{1-2}$ The vast majority of commercially available relative humidity sensors are based on ceramics ${ }^{2}$ (in particular, $\mathrm{Al}_{2} \mathrm{O}_{3}$, $\mathrm{Si}_{3} \mathrm{~N}_{4}$ ), metal oxides like $\mathrm{SnO}_{2}, \mathrm{GaN}$, polyelectrolytes, and conducting polymers. ${ }^{3-4}$ These sensors suffer from various drawbacks including poor sensitivity, slow response and recovery time, and narrow range of humidity detection. For this reason carbon nanomaterial-based humidity sensory materials, such as carbon nanotubes (CNTs), ${ }^{5}$ graphene oxide (GO), ${ }^{6-8}$ and reduced graphene oxide (rGO) ${ }^{9}$ are attracting a great attention in the last few years due to their excellent humidity-sensing capabilities resulting from the high surface-to-volume ratio making their physical and chemical properties extremely susceptible on their environment. Compared to traditional humidity sensors, those based on carbon-nanomaterials typically display higher mechanical and chemical stability, faster response, and high scalability. ${ }^{10} \mathrm{GO}$ and $\mathrm{rGO}$ in particular are under the spotlight due to their industrially scalable production, low-cost of the starting material, simplicity in their preparation and processability in green solvents, and high chemical stability, rendering them most suitable for applications in humidity sensing. ${ }^{6-9,}$, 11-13 The remarkable humidity sensing capability of GO originates from the presence of numerous hydrophilic functional groups on its surface (e.g., epoxide rings, hydroxyl and carboxyl groups) that can efficiently interact with water molecules from the environment. However, since the interactions between the water molecules and the aforementioned functional groups of GO are rather strong, GO-based humidity sensors suffer from large hysteresis and incoherent behavior over time. ${ }^{14}$ Moreover, their sensing performances are often characterized by slow response and recovery times. ${ }^{13,15-16}$ Furthermore, because of the poor conductivity of GO (often below $\left.1 \cdot 10^{-4} \mathrm{~S} / \mathrm{m}\right),{ }^{17}$ high potentials need to be applied in order to reach readable currents, thus resulting in increased power consumption. ${ }^{18-19}$ To overcome the poor 
conductivity of GO electrochemical impedance spectroscopy (EIS), which require a complex and expensive analyzing device, is also employed..$^{20}$ On the other hand, rGO is 6-7 orders of magnitude more conductive than $\mathrm{GO},{ }^{21}$ allowing the fabrication of power-efficient devices. ${ }^{22}$ Nonetheless, the main drawback of rGO is its hydrophobic nature; due to the elimination of the oxygen containing functional groups during the reduction processes of GO, its surface becomes hydrophobic, which causes a lower interaction with the water molecules and ultimately lower response to humidity. ${ }^{9}, 15$ Chemical functionalization of rGO repristinate some functional groups, which through their interaction with water may enhance rGO sensitivity. For instance Lee's et al. ${ }^{23}$ functionalized GO with terminating amines by using ethylenediamine, and demonstrated that the presence of amine groups improved the sensitivity towards humidity, but provoked unneglectable hysteresis due to the strong interaction between water molecules and the terminating amines.

Here we demonstrate that the sensitivity of rGO to relative humidity can be amplified through its chemical functionalization with hydrophilic moieties that by design specifically and reversibly interact at the supramolecular level with water molecules. We have focused our attention on triethylene glycol that can interact with water molecules forming weak, thus reversible, hydrogen bonding when compared to carbonyls, carboxylic acids and hydroxyls. ${ }^{24}$ To demonstrate our rational, a comparative test has been carried out also via the functionalization of GO with an aliphatic chains which, on the contrary, is more hydrophobic and features lower interactions with moisture. The performances towards humidity sensing of these two chemically modified rGO (rCMGO) are benchmarked with the pristine $\mathrm{rGO}$, and the mechanism at the basis of the different responses to humidity changes is elucidated with the aid of force field molecular dynamics simulations.

\section{EXPERIMENTAL METHODS}

Synthesis of
methoxyethoxy)ethoxy]ethylamine (NTEG)

All the reagents were bought from Sigma Aldrich and used without further purifications. The synthesis of NTEG consists in a three-step reaction: the first step is the synthesis of 2-[2-(2-Methoxyethoxy)ethoxy]ethoxy]ptoluenesulfonate (TEG-Tosylate) that was synthetized following the procedure reported by Ki Duk Park et al. ${ }^{25}$ The second and third steps of the reaction, aimed at the production of TEG-azide and finally NTEG, were performed following the procedure reported by Hyunkyu Kim et al. ${ }^{26}$ 1H NMR (400 MHz, CDCl3) $\delta 3.68-3.62(\mathrm{~m}, 6 \mathrm{H}), 3.55$ (dd, $\mathrm{J}=5.7,3.6 \mathrm{~Hz}, 2 \mathrm{H}), 3.51(\mathrm{t}, \mathrm{J}=5.2 \mathrm{~Hz}, 2 \mathrm{H}), 3.38(\mathrm{~s}, 3 \mathrm{H}), 2.86$ $(\mathrm{t}, \mathrm{J}=5.2 \mathrm{~Hz}, 2 \mathrm{H})$.

\section{Synthesis of Chemically Modified GO and prepara- tion of the devices}

$1.0 \mathrm{~mL}$ NTEG and $10 \mathrm{~mL}$ of $4 \mathrm{mg} / \mathrm{mL} \mathrm{GO}$ aqueous dispersion from Graphenea were added to $50 \mathrm{~mL}$ of ethanol $(\mathrm{EtOH})$ and the resulting mixture was refluxed for $12 \mathrm{~h}$. Then the mixture was cooled down and centrifuged 5 times in EtOH (6000 rpm, $10 \mathrm{~min}$ ), each time eliminating the supernatant to remove the excess of the unreacted NTEG. The solid was dispersed again in $80 \mathrm{~mL}$ of EtOH to obtain the CMGO-1 suspension $(0.5 \mathrm{mg} / \mathrm{mL})$. The same procedure was repeated adding $1 \mathrm{~mL}$ of decylamine instead of NTEG to prepare CMGO-2 and without the addition of any amine for GO. The suspensions of CMGOs and GO were diluted 10 times in EtOH and sonicated 10 minutes until stable and clear suspensions were formed. Suspensions of CMGOs with higher concentration exhibited limited stability and showed tendency to aggregate. These suspensions were spray-coated on freshly cleaned solid substrates (glass or $\mathrm{Si} / \mathrm{SiO} 2$ ) pre-heated at $100{ }^{\circ} \mathrm{C}$. A commercial airbrush gun for modelling and painting with a 0.3 $\mathrm{mm}$ needle and nozzle was used. The airbrush was supplied with compressed nitrogen at 1.5 bar. Typically microscope glass slides cut in $1.2 \times 1.2 \mathrm{~cm} 2$ squares were used. Finally, the samples were annealed under nitrogen at 240 ${ }^{\circ} \mathrm{C}$ for 10 minutes. Electrical contacts were fabricated by depositing two strips of $30 \mathrm{~nm}$ gold layer spaced by a 2 mm channel on the CMGO films with a thermal evaporator and a shadow mask.

\section{Characterization of the devices}

The performance of humidity sensors has been investigated by connecting the two electrical contacts to a source measure unit (SMU) Keithley 2636A. To measure the sensitivity, the response and recovery time a constant bias of 2 $\mathrm{V}$ was applied to the device and the current intensity was acquired for $30 \mathrm{~s}$, with a sampling frequency of $80 \mathrm{~Hz}$, while a pulsed flow of humid air was spilled on the sample. To do so a glass stopcock, connected to a balloon filled with humid air and fixed at $10 \mathrm{~cm}$ from the sample, was cyclically and manually opened. The values of initial humidity were included between 36 and $42 \% \mathrm{RH}$. The response and recovery times were calculated on the humidity pulses as the time interval between the $10 \%$ and the 90 $\%$ of the distance between the maximum response and the baseline. The values of response and recovery time reported in the text are the arithmetic mean of 5 different pulses. The calibration of the humidity response of the devices was performed including the devices connected to the SMU into a close chamber, in which humidity levels have been controlled by filling it with dry nitrogen. High levels of humidity in the chamber were obtained introducing into the chamber the $\mathrm{N}_{2}$ gas that was previously passed through a saturated aqueous solution of potassium sulfate $(\mathrm{RH}=98 \%)$. The values of the $\mathrm{RH}$ inside the chamber were measured with two independent commercial hygrometers. The response of the device was calibrated by reading the resistance and the corresponding $\mathrm{RH}$ values registered by the hygrometers, while the level of humidity inside the chamber was increased from $\mathrm{RH}=0 \%$ to $98 \%$ and viceversa (Figure S12a). The stability over time and the cyclability of the devices were gauged alternating for 10 times the $\mathrm{RH}$ in the chamber between 10 and $90 \%$ and recording the resistance. The calibration of the sensitivity to different vapors (ethanol, methanol, acetone and chloroform) were performed inside a glove-box by exposing alternatively the device to the environment of the glovesbox and to a close chamber saturated with vapors of the analytes, obtained by including in the chamber a small beaker with the dry liquid analyte and continuously measuring the resistance with the source-meter. The long-term stability of the rCMGO-1 device was evaluated including 
the device connected to the SMU in a sealed chamber in which a beaker containing a saturated solution of respectively $\mathrm{LiCl}, \mathrm{NaCl}$ and $\mathrm{K}_{2} \mathrm{SO}_{4}$ (Figure S12b). The high humidity and temperature test were performed by acquiring the calibration curve for humidity of rCMGO-1 device before and after the exposure of the device to the temperature of $80{ }^{\circ} \mathrm{C}$ and humidity of $79 \% \mathrm{RH}$. These conditions were obtained by placing the device and a small beaker containing a saturated solution of $\mathrm{KBr}$ inside a sealed container and this was placed into an oven at $80^{\circ} \mathrm{C}$ for 3 days. The vapor pressures of the saturated salt solution used were obtained from the article of Lewis Greenspan. ${ }^{27}$ The calibration of the rCMGO-1 sensor response to the temperature was performed by placing the device connected to the SMU inside a glove-box with water-free atmosphere, a thermocouple glued to the device was used to control the temperature. For measuring the response to temperature above RT the device was placed on top of an electrical heater with temperature control and the temperature of this was increased with a ramp of $5^{\circ} \mathrm{C} / \mathrm{min}$. The temperature read from the thermocouple and the resistance read from the SMU were acquired at $5{ }^{\circ} \mathrm{C}$ intervals until $90^{\circ} \mathrm{C}$. Likewise to acquire the response to temperature lower than RT the device was placed on top of a thermoelectric cooler and cooled to $-5{ }^{\circ} \mathrm{C}$ while the temperature and resistance were acquired. All the measurements were performed in a temperature range between 20 and $24^{\circ} \mathrm{C}$ unless differently reported.

\section{RESULTS AND DISCUSSION}

The surface of GO sheets possesses numerous reactive oxygen-rich functional groups such as hydroxyls, carboxylic acids and epoxides which can react with various organic molecules, including amines. ${ }^{28-30}$ Noteworthy, while the hydroxyls and carboxylic acid groups of GO need to be firstly activated to react with amines, ${ }^{31-33}$ ring opening reactions take place on epoxides even without any catalyst under mild experimental conditions. ${ }^{29-30,34}$ Because of this reason, the functionalization of GO with amines was conducted primarily by exploiting the epoxide ring opening reaction, yet not excluding the possibility of amide formation on some of the carboxylic groups. ${ }^{29-30,35-37}$ In order to minimize reagent waste, we decided to carry out the condensation reaction between GO and the amines in an ethanolic solution under reflux. GO was functionalized with two different primary amines, i.e. 2-[2-(2methoxyethoxy)ethoxy] ethylamine (NTEG) and decylamine. These two chemically modified GO are hereafter referred to as CMGO-1 and CMGO-2, respectively. The two amines have similar molecular length, yet the former is highly hydrophilic, while the latter is strongly hydrophobic. In parallel, we refluxed neat GO in ethanol, which served as reference sample and allowed comparison with CMGOs. The ethanolic suspensions of ca. $0.05 \mathrm{mg} / \mathrm{mL}$ of CMGOs and GO were processed into thin films on glass substrates by the spray-coating. Subsequently, GO films were partially reduced by a short $(10 \mathrm{~min})$ thermal annealing under nitrogen atmosphere at $240{ }^{\circ} \mathrm{C}$. The employed annealing conditions were chosen based on previously reported works, as they are sufficiently high to increase the conductivity of the GO-based materials, ${ }^{38-39}$ and mild enough to preserve the amine bond to CMGOs. ${ }^{40}$ Finally, top gold electrodes were patterned via sublimation in high vacuum through a shadow mask with electrodes distance of $5 \mathrm{~mm}$. A representation of the CMGOs synthesis and device preparation is portrayed in Figure 1.

The materials were characterized prior and after annealing to prove the successful functionalization and stability. $\mathrm{X}$-ray photoelectron spectroscopy (XPS) was used to gain quantitative insight into the chemical composition of hybrid materials by identifying the relevant chemical elements present in GO and CMGOs. The C 1s peak (Figure S1 in the Supporting Information) of CMGO-1 and CMGO-2 exhibits a hump at $285.9 \mathrm{eV}$ which indicate the $\mathrm{C}-\mathrm{N}$ bond formation, ${ }^{41-42}$ as evidence in the deconvoluted peaks. This hump is also present in rCMGO-1 and rCMGO-2 (yet with a lower intensity for rCMGO-2), while is completely absent in rGO (Figure 2a and Figure S1 in the Supporting Infor-

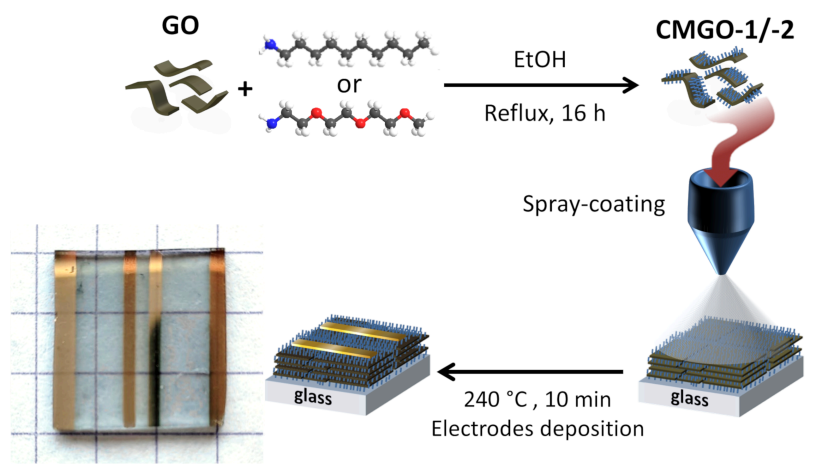

Figure 1. Schematic representation of the reaction of GO with either NTEG or decylamine, and device preparation. Bottom-left: photo of the actual sensor (lateral size: $1.2 \mathrm{~cm}$ ). The underlying square pattern serves to demonstrate the optical transparence of the device.

mation).

Simultaneously, the spectral features associated to the various $\mathrm{C}-\mathrm{O}$ bonds are drastically less intense in all the reduced species, in accordance with the effects of the thermal reduction of G0.43 As expected, $\mathrm{N}$ 1s peaks are negligible in GO and rGO, while in CMGOs and rCMGOs they are strong (Figure $2 \mathrm{~b}$ and $\mathrm{S} 2$ in the Supporting Information). XPS can also provide semi-quantitative information concerning the composition: 44 from the atomic relative abundance of carbon, nitrogen and oxygen obtained by fitting the XPS survey spectra showed in Table S4 of the Supporting Information we can assess the degree of functionalization, which was found to be comparable for the two systems. The preservation of the chemical functionalization under the annealing conditions combined with a reduction of the GO scaffold evidenced by the loss of hydroxyls moieties are highlighted by TGA analysis (see Supporting Information for details). The partial reduction of the GO scaffold is also evidenced by the decrease in transmittance of the films (Figure S6 of the Supporting Information); 45 it is worth noting that the final devices are rather transparent (transmittance at $550 \mathrm{~nm}$ of $65 \pm 5 \%$ ).

The successful functionalization of GO was also confirmed by the IR spectroscopy attenuated total reflection 
(FTIR-ATR); the spectrum of CMGO-1 and CMGO-2 (Figure 2c) exhibits characteristic peaks which are absent in GO. In particular, they both show the typical peak of the stretching of $\mathrm{CH}_{2}$ groups at ca. $2850 \mathrm{~cm}^{-1}$, which is present in the two molecular backbone (detailed assignment of the peaks is reported in the Supporting Information). Raman spectroscopy is an important technique to evaluate the presence of defects and disorder in graphene and GO, in particular through the analysis of the ratio between the intensities of $D$ and $G$ peaks $\left(I_{D} / I_{G}\right)$ that are the main spectral features of graphene related materials. In the present case, this ratio was found being superior in CMGOs and reduced CMGOs compared to GO and rGO samples, which originates from the higher defectiveness of CMGOs as a result of the chemical functionalization. ${ }^{46}$ Conversely, such ratio decreases after thermal reduction for all samples, due to the growth of $\mathrm{sp}^{2}$-hybridized regions (Figure S5 in the Supporting Information).

The deposited and annealed films were also investigated through Scanning Electron Microscopy (SEM) and Atomic Force Microscopy (AFM) to evaluate the morphology, roughness, and film thickness. The SEM images are reported in Figure S8 of the Supporting Information. The films appear uniform and continuous all over the substrate. All the samples look quite similar, with flat surfaces in which the GO flakes stuck one over the other in a layered motif. However, compared to $\mathrm{rGO}$, both $\mathrm{rCMGO}-1$ and rCMGO-2 display more wrinkles and jutting edges. A more detailed analysis on samples morphology is given by AFM, which provides the topography of the surface. The surface roughness (Rq) calculated from the AFM images (Figure S9 of the Supporting Information) follows the trend rGO (4.49 $\mathrm{nm})<$ rCMGO-1 (7.21 nm) < rCMGO-2 (14.29 nm). We ascribe such a difference to the diverse dispersibility of GO, CMGO-1 and CMGO-2 in ethanol which follows the same trend, being GO the most dispersible. Even though the suspension was sonicated for 15 minutes before the coating, small aggregates of CMGO-1 and CMGO-2 not visible to naked eye could still be present. The film thickness was also evaluated by AFM, by scratching the film surfaces to expose the substrate and imaging the scratch edge. The results as well as the height profiles are reported in Figure S10 of the Supporting Information. In the case of rCMGO-2 for simplicity we imaged a small hole present in the surface of the film. The average film thickness was $23.7 \mathrm{~nm}$ for rCMGO-1, $22.9 \mathrm{~nm}$ for rGO, and $39.5 \mathrm{~nm}$ for rCMGO-2.

The hydrophilicity of the different spray-coated films was quantified by water contact angle (C.A.) measurements (Figure S11 of the Supporting Information): rCMGO1 resulted the most hydrophilic (C.A. $=63.1^{\circ} \pm 3.4^{\circ}$ ), while rGO and rCMGO-2 were found being more hydrophobic (with a C.A of $87.3^{\circ} \pm 1.7^{\circ}$ and $92.4^{\circ} \pm 1.0^{\circ}$, respectively). The X-ray diffraction (XRD) spectra recorded in situ by systematically changing the $\mathrm{RH}$ provided information about the structure and the stability of rCMGOs and rGO films under humidity exposure (Figure 2d-f). They revealed that at $\mathrm{RH}$ $=0 \%$ the 001 peak of rCMGOs shifts towards a smaller $2 \Theta$ angle compared to rGO. The corresponding interlayer distance calculated following the Bragg's law changes from $4.82 \AA$ of rGO to $8.38 \AA$ of rCMGO- 1 and $8.37 \AA$ of rCMGO-2, indicating that the presence of TEG and decylamine chains imposes higher interflake distances in rCMGOs. Furthermore, a subtle change in the crystallinity of the materials is observed upon exposure to humidity: the spacing between rCMGO- 1 and rGO layers increases by only $0.16 \AA$ when $\mathrm{RH}$ increases from $0 \%$ to $80 \%$ (Figure $2 \mathrm{~d}$,f) whereas such a
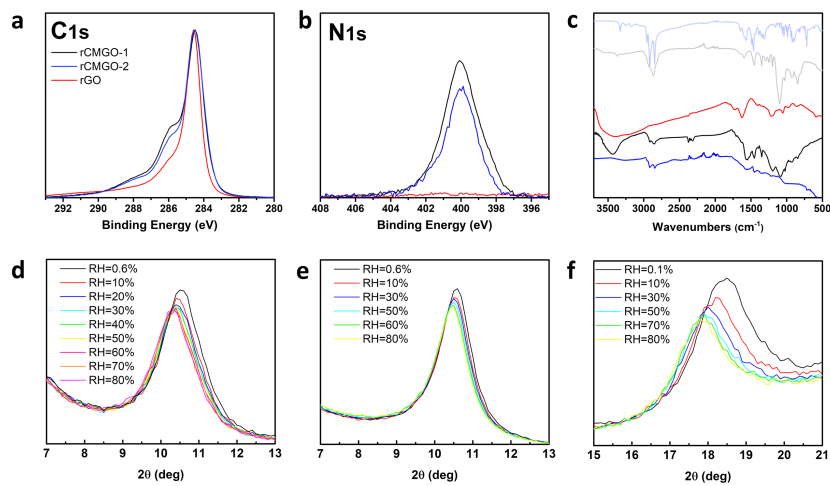

Figure 2. XPS spectra of C1s peak (a) and N1s peak (b) of rCMGO-1 (black), rCMGO-2 (blue) and rGO (red). c) FTIR-ATR of CMGO-1 (black), CMGO-2 (blue) and GO (red), decylamine (light blue) and NTEG (gray). Variation of the XRD patterns upon exposure to humidity of the (d) rCMGO-1, (e) rCMGO-2, and (f) rGO films.

small increase is negligible for $\mathrm{rCMGO}-2$ with an interlayer spacing enhancement of only 0.09 Å (Figure 2e). Noteworthy, the expansion of rGO films is similar to those of rCMGO-1 because, despite the higher hydrophilicity of rCMGO-1 compared to rGO, in the former the water molecules have less free space between the layers due to the steric effect of the dangling molecules. Such interlayer expansion is minimal for rCMGO-2 because of both its high hydrophobicity and few empty spaces between layers. The XRD of the not reduced films and a more detailed analysis are reported in the Supporting Information.

Once the successful functionalization was confirmed, different electrical measurements were performed on the devices fabricated from the three reduced materials to gain quantitative insight into the key performance indicators of the humidity sensor, i.e. sensitivity, response and recovery time, reversibility, long term stability and selectivity. The response to $\Delta \mathrm{RH}$ was defined as the ratio between the change in resistance $\Delta R$ and the initial resistance $R_{0}$ in percentage upon exposure of the devices to humidity or to vapor whereas the sensitivity as the response over a specific variation in relative humidity. Firstly, the response of the devices as a function of time was measured while sending a pulsed flow of humid air over the sample while applying a constant bias of $2 \mathrm{~V}$, and measuring the electrical current to evaluate the response intensity and stability. Figure 3a portrays the response of rCMGO-1 and rCMGO-2 and $\mathrm{rGO}$ once exposed to a series of 100 pulses. It shows that the response is almost constant for the rCMGO-1 device, even after 100 pulses and the small variations are more likely caused by the rough approach employed to provide the humid pulses. In addition the baseline of this device is particularly stable; in fact by keeping the humidity constant the resistance of the device shows fluctuations below the $0.01 \%$ (Figure S14 of the Supporting Information). Conversely, the rGO sensor exhibits a more instable behavior(Figure 3a). In fact, the intensity of the re- 
sponse varies from a maximum of $1 \%$ to just the $0.07 \%$, and it becomes progressively weaker after several pulses. Such a trend may be due to the progressive saturation of the oxygen containing moieties of the rGO with water molecules. Furthermore, instability of the baseline has been monitored, revealing a dramatic increase during the first 10 pulses, then stabilizing and finally decreasing slowly to the initial values. On the other hand, the sensor based on rCMGO-2 shows a more stable response than the one comprising rGO; however, its baseline reveals a similar instability to rGO, with an initial rapid increase followed by a more stable behavior. To monitor the response to single humidity pulses the devices were also subjected to a lower frequency of pulses (Figure $3 \mathrm{~b}$ ). The rCMGO-1 sensor displays the highest sensitivity (being almost three and seven times greater than rGO and rCMGO-2, respectively), a constant and a completely reversible response. This is not the case of rGO and rCMGO-2, whose baselines increase over time because of a not complete recovery. Figure 3c shows magnifications of single pulses of humidity, from which the response and recovery times of the devices were calculated. Significantly, rCMGO-1 device responds in just $25 \mathrm{~ms}$ and fully recovers in $127 \mathrm{~ms}$. Conversely, the rGO sensor exhibits a 5-fold increase in the response and a not complete recovery which is 15 times longer, and the rCMGO-2 devices exhibits a response and recovery time respectively 4 and 6 times longer.
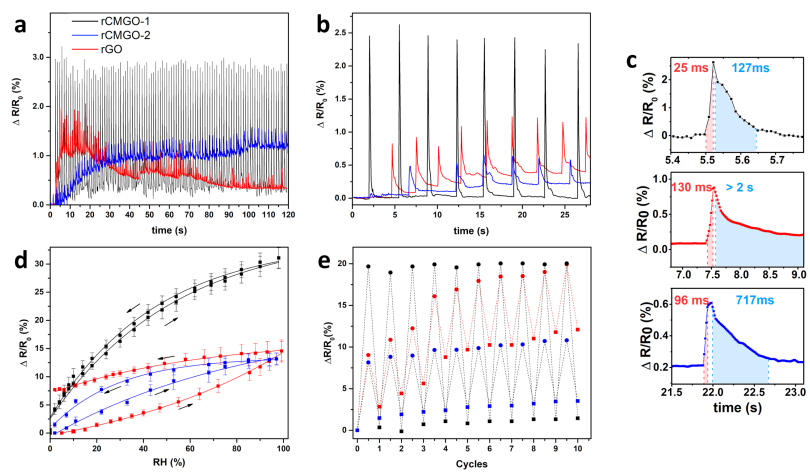

Figure 3. Variation of the resistance expressed in \% (response) of the devices rCMGO-1 (black), rCMGO-2 (blue) and rGO (red). a) Response when the devices are exposed to 100 pulses of humid air during the application of a constant bias of $2 \mathrm{~V}$. b) Response of the same devices under a lower number of pulses and c) magnification of the response to a single pulse with reported response time (in red) and recovery time (in light blue), which correspond to the highlighted regions (from top to bottom rCMGO-1, rGO and rCMGO-2). d) Response in function of the measured RH (\%) when the humidity is gradually increased and decreased. e) Response of the devices upon cycling the RH between $10 \%$ (squares) and $90 \%$ (circles) 10 times.

For comparison the commercial sensor Sensirion SHT31 when exposed to a similar pulse of humid air shows a response and recovery time of 0.5 and 3 s respectively (Figure S15 of the Supporting Information). The response of the three devices to different humidity levels was calibrated by measuring the electrical output of the devices while increasing the relative humidity from $2 \%$ to $97 \%$ and then decreasing it (Figure 3d). It is possible to observe that the sensitivity of the $\mathrm{rCMGO}-1$ sensor over the entire range of $\mathrm{RH}$ is around $30 \%$ and the response data can be perfectly fitted with a negative exponential curve. Noteworthy, there is no threshold of the response and that the maximum sensitivity is observed for low RH values. Furthermore, the device exhibited low hysteresis $(1.1 \%)$. On the contrary, the rGO and rCMGO-2 samples showed a lower sensitivity, being less than half compared to rCMGO-1. Additionally, the response of rGO has a different trend upon increasing the $\mathrm{RH}$ respect to the $\mathrm{rCMGO}-1$, and it can be fitted by a positive exponential. However, in the return curve the trend is again a negative exponential, which results in a large hysteresis (around $67 \%$ of $\Delta R / R_{0}$ ). Such behavior suggests that a high resistance is retained even after the humidity level has decreased because of a more difficult release of the water molecules absorbed in the rGO sample.

The rCMGO-2 sensor exhibits an intermediate behavior, with a response that can be fitted with a negative exponential in both direction and a lower, but still quite important hysteresis (21\%). Significantly, the r-CMGO-1 sensor retains a stable response even after 10 cycles between 10 and $90 \%$ of $\mathrm{RH}$; conversely, the response of rGO and rCMGO-2 is not stable after different cycles since the devices showed a continuous increase in resistance (Figure 3e). The devices were also cycled between low and high humidity by plotting continuously the change in resistance over time. These results are reported in Figure S16 of the Supporting Information and confirm the higher stability and sensitivity of rCMGO-1.

The resistance of $\mathrm{rCMGO}-1$ sensor remained nearly constant (changes $<1.5 \%$ ) even when the sample was left at the extreme conditions of $\mathrm{RH}=11 \%$ and $\mathrm{RH}=98 \%$ for 10 days (Figure S17a of the Supporting Information). To assess further the robustness of our device, it was also exposed to the harsh environment of $80^{\circ} \mathrm{C}$ and $80 \% \mathrm{RH}$ for 3 days. The response of rCMGO-1 to different levels of humidity was acquired before and after the extreme environment test and showed a small decrease in the resistance of $3.4 \%$ after the test (Figure S17b of the Supporting Information). However, the response remained constant $(0.7 \%$ increase), thus the $\mathrm{rCMGO}-1$ sensor can be exposed to extreme conditions without requiring recalibration. The effect of temperature on the resistance of rCMGO-1 was also evaluated, by keeping constant the humidity at $0 \% \mathrm{RH}$ and varying the temperature from $0{ }^{\circ} \mathrm{C}$ to $80^{\circ} \mathrm{C}$ (Figure S18 of the supporting Information). The resistance decreases when the temperature increases and the points can be well fitted by an exponential equation in the form $R=R_{0} \exp (k / T)$ indicating a thermally activated band-like conductivity in the sample. ${ }^{47}$

The cross-sensitivity of the devices to vapors of volatile organic compounds (VOCs) was also evaluated. In particular, the sensitivity towards dehydrated ethanol, methanol, acetone and chloroform was tested by recording the response of the devices while exposing them subsequently to nitrogen gas and to equilibrium vapor pressure of the selected VOCs for two cycles. The results are plotted in Figure S19 of the Supporting Information and the first cycle is reiterated in Figure 4 (a-c). It unambiguously shows that the response of $\mathrm{rCMGO}-1$ to all the VOCs is the lowest. In order to compare the sensitivity of all sensory 
materials to the different VOCs and to RH, we considered that all the analyzed VOCs are more volatile than water,

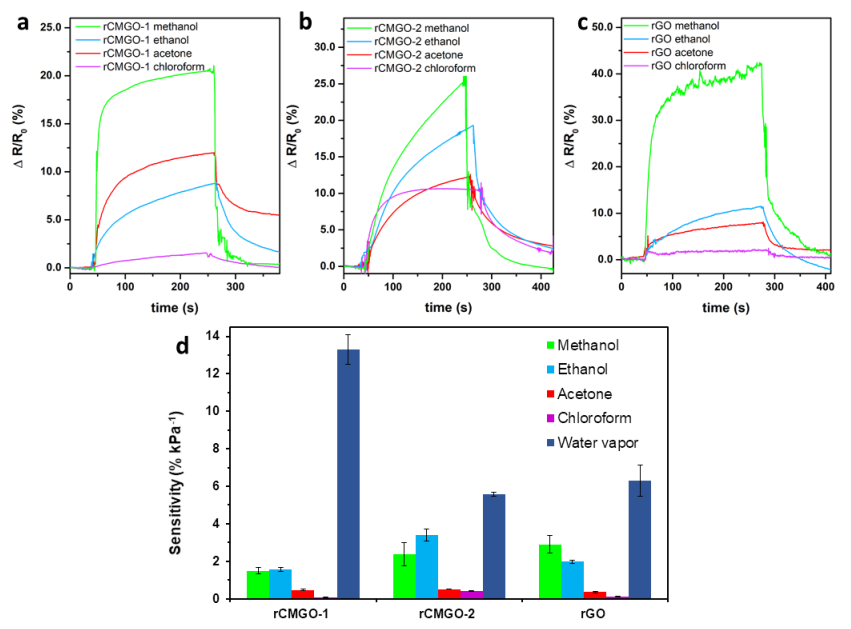

Figure 4. Selectivity response in function of time when a) rCMGO-1, b) rCMGO-2 and c) rGO are exposed alternatively to nitrogen and to saturated vapours of methanol (green line), ethanol (blue line), acetone (red line) and chloroform (purple line). d) Sensitivity of the devices $\left(\% \mathrm{kPa}^{-1}\right)$ to the same vapours and to humidity ( $100 \% \mathrm{RH})$ obtained dividing the maximum response to the analytes by its saturation vapor pressure at $20^{\circ} \mathrm{C}$ expressed in $\mathrm{kPa}$.

thus can reach higher concentration at saturation. For this reason, the responses of the devices to the saturated vapors of the analytes were normalized dividing them by the saturation vapor pressure of each analyte at $20^{\circ} \mathrm{C}$, expressed in $\mathrm{kPa}$. The results are reported in Table $\mathrm{S} 2$ of the Supporting Information and graphically in Figure 4d. All the sensors exhibit the highest response to methanol and ethanol, which can both donate and accept hydrogen bond, thus being the most similar to water. Furthermore, ethanol and methanol dipole moments are also similar to the one of water, which can explain the relatively high sensitivity to these vapors. In particular, rCMGO-2 displays the highest response to ethanol $\left(3.19 \% \mathrm{kPa}^{-1}\right)$, whereas $\mathrm{rGO}$ shows the highest response to methanol $\left(2.81 \% \mathrm{kPa}^{-1}\right)$. The sensitivity to acetone, which can only accept hydrogen bonds, is lower for all the devices and the sensitivity to chloroform is comprehensibly even lower, being unable to form hydrogen bonds. However, while rCMGO-1 has negligible sensitivity to chloroform $\left(0.07 \% \mathrm{kPa}^{-1}\right)$, the aliphatic chains which decorate the surface of $\mathrm{rCMGO}-2$ determine a higher affinity for chloroform, resulting in a $0.43 \% \mathrm{kPa}^{-1}$ response to its vapor. rCMGO-2 also shows an increase in the response for high concentrations of methanol and ethanol, which may be due to a disruption of the alkyl chains packing at high concentration of these vapors. Interestingly, rCMGO-1 alongside being the most sensitive to humidity, it possesses also the lowest cross sensitivity to each one of the analyzed VOCs. In particular, rCMGO- 1 is at least 9 times more sensitive to water than to any other analyte.

The key performance indicators of rCMGO- 1 were also benchmarked against commercial relative humidity sensors and to the state-of-the-art rGO and GO based sensors that are reported in literature (Table 1). It is clear that rCMGO-1 outperforms most of the commercial sensors that show similar performances in terms of sensitivity, but slower response and recovery while it performs better than all the rGO based sensors for sensitivity, response speed and recovery. Only GO sensors seem to have a higher sensitivity at the expense of the conductivity of the material, that on the one hand obliges to operate the device with high frequency alternating currents, thus complicating the measurement and on the other hand restricts the sensing range because of the insulating behavior of GO at low humidity and low reversibility when GO is exposed to high humidity. ${ }^{14}$

Table 1. Comparison of the performances of selected commercial humidity sensors and those based on G0/rGO which are described in the literature.

\begin{tabular}{|c|c|c|c|c|c|c|}
\hline Type & Sensor & $\begin{array}{l}\text { Sensitivity } \\
\%\left(\% \mathrm{RH}^{-1}\right)\end{array}$ & Hysteresis & Response time & $\begin{array}{l}\text { Recovery } \\
\text { time }\end{array}$ & $\begin{array}{l}\text { Humidity } \\
\text { range }(\% \mathrm{RH})\end{array}$ \\
\hline C (commercial) & IST P14-W & $0.17 \%$ & $<1.5 \%$ & $5 \mathrm{~s}$ & & $0-100 \%$ \\
\hline C (commercial) & $\begin{array}{l}\text { E+E Elektronik } \\
\text { HCT01 }\end{array}$ & $0.35 \%$ & $<1.85 \%$ & $6 s$ & & $0-100 \%$ \\
\hline C (commercial) & Sensirion SHT10 & $6 \%$ & $1 \%$ & $6 s$ & & $0-100 \%$ \\
\hline $\mathrm{R}$ (commercial) & Sensirion SHT31 & $8 \%$ & $0.8 \%$ & $8 \mathrm{~s}$ & & $0-100 \%$ \\
\hline C (article) & GO 18 & $472 \%$ & & $10 \mathrm{~s}$ & $41 \mathrm{~s}$ & $15-95 \%$ \\
\hline $\mathrm{Z}$ (article) & $\mathrm{GO}^{6}$ & $25 \%$ & & $20 \mathrm{~ms}$ & $30 \mathrm{~ms}$ & $40-80 \%$ \\
\hline R (article) & dGO-PVA 16 & $11.5 \%$ & & $>60 \mathrm{~s}$ & $>60 \mathrm{~s}$ & $40-95 \%$ \\
\hline R (article) & $\mathrm{rGO}^{9}$ & $0.04 \%$ & & & & $40-95 \%$ \\
\hline R (article) & GO-aniline 19 & $43 \%$ & & $50 \mathrm{~ms}$ & $50 \mathrm{~ms}$ & $10-90 \%$ \\
\hline R (article) & PDDA/rGO 48 & $0.31 \%$ & $2 \%$ & $94 \mathrm{~s}$ & $133 \mathrm{~s}$ & $11-97 \%$ \\
\hline R (this work) & rCMGO-1 & $0.33 \%$ & $1.1 \%$ & $25 \mathrm{~ms}$ & $127 \mathrm{~ms}$ & $0-100 \%$ \\
\hline
\end{tabular}


R : resistive, C : capacitive, Z : impedance, dGO : poly(dopamine) coated GO, PVA : poly(vinyl alcohol), PDDA: poly(diallylimethyammonium

To gain further insight into the nature of the interaction between water molecules and rGO/rCMGO surfaces and reveal the origin of the different response of CMGOs, force field MD simulations were performed in the presence of explicit water molecules (see SI for details), on rGO and rCMGO-1 layers built according to the experimental atomic composition and surface coverage (for rCMGO-1). To ease a quantitative comparison between the three surfaces, a model for rCMGO-2 was constructed by replicating the surface density of rCMGO-1. Compared to rCMGO-1, the density of side chains is comparable in the measured rCMGO-2 samples, hence we also built two additional surfaces (hitherto referred as $\mathrm{CCMGO}-1 \mathrm{H}$ and $\mathrm{rCMGO}-2 \mathrm{H}$ ) with half the surface coverage of rCMGO-1. All of these surfaces were then immersed in a water box and subjected to MD preparation and sampling runs (see SI for details). Irrespective of the coverage, the calculated Pair Interaction (PI) energies between water molecules and the (functionalized) layers follow the trend $\operatorname{rCMGO}-1(\mathrm{H})>\mathrm{rGO}>$ rCMGO-2(H), in line with the changes in hydrophobic/hydrophilic character of the surface and explaining the trend in sensitivity of the three materials (Figure 5a).
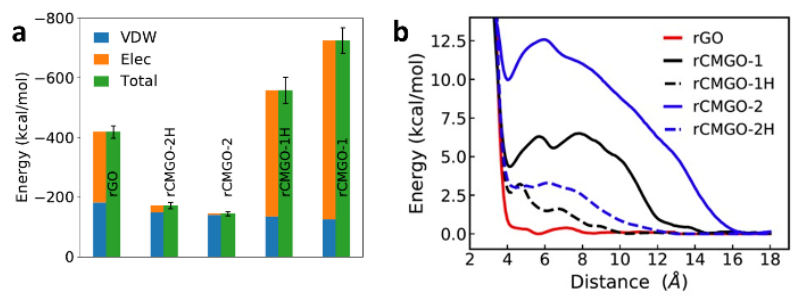

Figure 5. a): Average pair interaction energy between water molecules and respective rGO/rCMGO surfaces and right $b$ ): PMF energy profile of water molecules with rGO (red), rCMGO-1/rCMGO-1H (black) and rCMGO-2/rCMGO-2H (blue) surfaces. VDW, Elec energies correspond to van der Walls and electrostatic contributions to the total (Total) energies.

Subsequently, Potential of Mean Force (PMF) profiles of water molecule (selected at random) with respective rGO/rCMGOs surfaces were computed, as reported in Figure $5 b$. The PMF free energy profile for rGO is very flat (likely because the enthalpic gain due to interaction between water molecules and the rGO layer is compensated by entropic losses as the molecules land on the surface), suggesting that water molecules can adsorb/desorb freely from the rGO surface. While the free energy shows a more pronounced surge in proximity to the surface in rCMGO-2 compared to rCMG0-1, both profiles feature a local minimum at around $4 \AA$. This minimum, which is absent for rGO, is mostly associated with a 'mechanical' trapping of water molecules in nano-pockets formed around the anchoring groups. This is borne out by results obtained for the half-coverage simulations showing a repulsive PMF profile all the way from 2D-surface to bulk water in CMGO$2 \mathrm{H}$ (and to a lesser extent in CMGO-1H).

Most importantly, at both low and high coverage, the rCHGO-1(H) surfaces equipped with glycolated side chains are characterized by an additional local minimum on the PMF curve at around $6 \AA$, which results from interaction between water molecules and the NTEG-oxygen atoms. As a matter of fact, this minimum is completely absent for the decylamine-decorated rCMGO-2(H) surfaces. The picture that emerges from these calculations is that the favorable interactions between water and the glycolated chains grafted on the surface enable a dynamic equilibrium in rCMGO-1, where the water molecules can be stored into metastable configurations at a distance from the 2D graphene layer $(\sim 6 \AA)$ that is sufficiently close to perturb charge transport while still being able to desorb through crossing a small energy barrier $(\sim 0.2-0.8 \mathrm{kcal} / \mathrm{mol}$ for rCMGO-1(H) surfaces) and thereby switching the system back to its original electrical response. We furthermore speculate that the instability and hysteresis behavior observed experimentally for $\mathrm{rGO}$ and $\mathrm{rCMGO}-2$ arise because of the completely flat surface energy potential in the former case (hence it is impossible to desorb all water molecules from the surface) and local inhomogeneity in the surface coverage density in the latter case (with trapping of water molecules at van der Waals contact with the surface in densely grafted regions). Furthermore, the orientation of selected water molecule in the micro-states at distance 4 and $6 \AA$ were computed (see SI for details). While rCMGO-1 shows a specific orientation of the water molecules in the two discrete micro-states, rCMGO-2 exhibits partially defined orientation of water molecules only at distance $4 \AA$, while rGO shows an isotropic distribution of the orientations of the water molecule in the quasicontinuous ensemble of microstates. The set of discrete microstates with low energy barriers and a constrained orientation of water molecule at the micro-states in rCMGO-1 leads to the higher and faster response of this material.

\section{CONCLUSION}

In summary, we have investigated the effect of the hydrophilicity of rGO and its chemically modified analogues over the humidity sensing capabilities. In particular, we produced two novel hydrophilic and hydrophobic materials based on the chemical modification of rGO with triethylene glycol and decane derivatives, respectively. These two materials and rGO were processed in thin and transparent resistive-type humidity sensors and exposed to humidity. We proved that the device comprising the most hydrophilic material, namely the glycolated rCMG0-1, possesses the better sensing performances towards humidity. This device shows an interesting positive response with a remarkable sensitivity (up to $31 \%$ when the RH is shifted from 2 to $97 \%$ ), high stability of the signal after $100 \mathrm{hu}-$ mid pulses, complete reversibility, fast response and recovery time (respectively $25 \mathrm{~ms}$ and $127 \mathrm{~ms}$ ), and longterm stability thereby outperforming many of the commercial devices and other rGO based resistive type devices. Our sensor displays also high selectivity to humidity when compared to representative small polar organic molecules. Conversely, the alkyl decorated rCMGO-2 and the nonfunctionalized rGO exhibit worse sensitivity, longer response and recovery times and instable behavior. The use 
of humidity dependent XRD and Force Field MD simulations made it possible to unravel the physical origin of the better performances of rCMGO-1 based devices. While, the functional groups present on the rGO surface have low impact on the structural changes of the materials when they are exposed to a humid environment, the presence of chemically attached molecules can favor or disfavor the interaction of water molecules with the graphene surface, leading to a different electronic behavior. Overall, we have demonstrated that the ad hoc chemical functionalization of GO with suitably designed molecules enables to reach highest performances in humidity sensing and ensures the highest selectivity to water molecules. We can anticipate that the analogous functionalization of GO and other 2D materials with the specific receptor of the analyte of choice, wisely chosen by exploiting the well-established library provided by supramolecular chemistry, will make it possible to fabricate highly performing chemical sensors of the analyte of interest. Finally, we believe that our sensor matches all the technological requirements to be integrated in portable, low-cost and low-consumption devices which potentially can be supported on flexible and transparent substrates.

\section{ASSOCIATED CONTENT}

Supporting Information. Graphs of the materials characterization, detailed description of Infrared spectroscopy analysis, Thermogravimetric analysis, estimation of the functionalization grade by XPS, interlayer spacing vs relative humidity, characterization of the humidity sensing device, Molecular Dynamic, and instrumental methods.

This material is available free of charge via the Internet at http://pubs.acs.org.

\section{AUTHOR INFORMATION}

\section{Corresponding Author}

A. Ciesielski, P. Samorì - Institut de Science et d'Ingénierie Supramoléculaires ,Université de Strasbourg, CNRS,8 alleé Gaspard Monge, 67000 Strasbourg, France;

Email: ciesielski@unistra.fr, samori@unistra.fr

D. Beljonne - CMN, Université de Mons, Place du Parc 20, 7000 Mons, Belgium

Email: david.beljonne@umons.ac.be

\section{Author Contributions}

The manuscript was written through contributions of all authors. All authors have given approval to the final version of the manuscript.

\section{ACKNOWLEDGMENT}

We acknowledge financial support from the European Commission through the ERC project SUPRA2DMAT (GA-833707), the Graphene Flagship Core 2 project (GA-785219), the MERA.NET project MODIGLIANI, the Agence Nationale de la Recherche through the Labex projects CSC (ANR-10-LABX$0026 \mathrm{CSC}$ ) and NIE (ANR-11-LABX-0058 NIE) within the Investissement d'Avenir program (ANR-10-120 IDEX-0002-02), the International Center for Frontier Research in Chemistry (icFRC). The work in Mons is supported by FNRS/F.R.S. D.B. is FNRS research director.

\section{REFERENCES}

1. Ruiz-Garcia, L.; Lunadei, L.; Barreiro, P.; Robla, I., A Review of Wireless Sensor Technologies and Applications in Agriculture and Food Industry: State of the Art and Current Trends. Sens. 2009, 9 (6), 4728.

2. Chen, Z.; Lu, C., Humidity Sensors: A Review of Materials and Mechanisms. Sens. Lett. 2005, 3 (4), 274-295.

3. Kuang, Q.; Lao, C.; Wang, Z. L.; Xie, Z.; Zheng, L., HighSensitivity Humidity Sensor Based on a Single SnO2 Nanowire. J. Am. Chem. Soc. 2007, 129 (19), 6070-6071.

4. Sakai, Y.; Sadaoka, Y.; Matsuguchi, M., Humidity Sensors Based on Polymer Thin Films. Sens. Actuators, B 1996, 35 (1), 8590.

5. Chen, H.-W.; Wu, R.-J.; Chan, K.-H.; Sun, Y.-L.; Su, P.-G., The Application of CNT/Nafion Composite Material to Low Humidity Sensing Measurement. Sens. Actuators, B 2005, 104 (1), 80-84.

6. Borini, S.; White, R.; Wei, D.; Astley, M.; Haque, S.; Spigone, E.; Harris, N.; Kivioja, J.; Ryhänen, T., Ultrafast Graphene Oxide Humidity Sensors. ACS Nano 2013, 7 (12), 11166-11173.

7. Jung, I.; Dikin, D.; Park, S.; Cai, W.; Mielke, S. L.; Ruoff, R. S., Effect of Water Vapor on Electrical Properties of Individual Reduced Graphene Oxide Sheets. J. Phys. Chem. C 2008, 112 (51), 20264-20268

8. Yao, Y.; Chen, X.; Zhu, J.; Zeng, B.; Wu, Z.; Li, X., The Effect of Ambient Humidity on the Electrical Properties of Graphene Oxide Films. Nanoscale Res. Lett. 2012, 7 (1), 363-370.

9. Tai, Y.; Bera, T. K.; Lubineau, G.; Yang, Z., Combining the Converse Humidity/Resistance Response Behaviors of rGO Films for Flexible Logic Devices. J. Mater. Chem. C 2017, 5 (15), 38483854 .

10. Yuan, W.; Shi, G., Graphene -Based Gas Sensors. J. Mater. Chem. A 2013, 1 (35), 10078-10091.

11. Rathi, K.; Pal, K., Impact of Doping on GO: Fast Response-Recovery Humidity Sensor. ACS Omega 2017, 2 (3), 842-851.

12. Teradal, N. L.; Marx, S.; Morag, A.; Jelinek, R., Porous Graphene Oxide Chemi-capacitor Vapor Sensor Array. J. Mater. Chem. C 2017, 5 (5), 1128-1135.

13. Anichini, C.; Czepa, W.; Pakulski, D.; Aliprandi, A.; Ciesielski, A.; Samorì, P., Chemical Sensing with 2D Materials. Chem. Soc. Rev. 2018, 47 (13), 4860-4908.

14. Naik, G.; Krishnaswamy, S., Room-Temperature Humidity Sensing Using Graphene Oxide Thin Films. Graphene 2016, 5, 1-13.

15. Papamatthaiou, S.; Argyropoulos, D. P.; Farmakis, F.; Masurkar, A.; Alexandrou, K.; Kymissis, I.; Georgoulas, N., The Effect of Thermal Reduction and Film Thickness on fast Response Transparent Graphene Oxide Humidity Sensors. Procedia Eng. 2016, 168, 301-304.

16. Hwang, S. H.; Kang, D.; Ruoff, R. S.; Shin, H. S.; Park, Y. B., Poly(vinyl alcohol) Reinforced and Toughened with Poly(dopamine)-Treated Graphene Oxide, and Its Use for Humidity Sensing. ACS Nano 2014, 8 (7), 6739-6747.

17. Gilje, S.; Han, S.; Wang, M.; Wang, K. L.; Kaner, R. B., A Chemical Route to Graphene for Device Applications. Nano Lett. 2007, 7 (11), 3394-3398.

18. Bi, H. C.; Yin, K. B.; Xie, X.; Ji, J.; Wan, S.; Sun, L. T.; Terrones, M.; Dresselhaus, M. S., Ultrahigh humidity sensitivity of graphene oxide. Scientific Reports 2013, 3, 2714

19. Tang, M.; Zhang, C.; Zhang, J. Y.; Zhao, Q. L.; Hou, Z. L.; Zhan, K. T., Ultrafast-Response Humidity Sensor with High Humidity Durability Based on a Freestanding Film of Graphene Oxide Supramolecular. Phys. Status Solidi A 2020, 217 (2).

20. Lv, C.; Hu, C.; Luo, J.; Liu, S.; Qiao, Y.; Zhang, Z.; Song, J.; Shi, Y.; Cai, J.; Watanabe, A., Recent Advances in Graphene-Based Humidity Sensors. Nanomaterials 2019, 9 (3), 422.

21. Kim, S. G.; Lee, S. S.; Lee, E.; Yoon, J.; Lee, H. S., Kinetics of Hydrazine Reduction of Thin Films of Graphene Oxide and the 
Determination of Activation Energy by the Measurement of Electrical Conductivity. RSC Adv. 2015, 5 (124), 102567-102573.

22. Phan, D. T.; Chung, G. S., Effects of Rapid Thermal Annealing on Humidity Sensor Based on Graphene Oxide Thin Films. Sens. Actuator B-Chem. 2015, 220, 1050-1055.

23. Lee, S.-W.; Choi, B. I.; Kim, J. C.; Woo, S.-B.; Kim, Y.-G.; Kwon, S.; Yoo, J.; Seo, Y.-S., Sorption/desorption hysteresis of thinfilm humidity sensors based on graphene oxide and its derivative. Sens. Actuators, B 2016, 237, 575-580.

24. Steiner, T., Effect of Acceptor Strength on C-H...O Hydrogen-Bond Lengths as Revealed by and Quantified from Crystallographic Data. J. Chem. Soc., Chem. Commun. 1994, (20), 2341-2342.

25. Park, K. D.; Liu, R.; Kohn, H., Useful Tools for Biomolecule Isolation, Detection, and Identification: Acylhydrazone-Based Cleavable Linkers. Chem. Biol. 2009, 16 (7), 763-772.

26. Kim, H.; Kang, Y. J.; Jeong, E. S.; Kang, S.; Kim, K. T., Glucose-Responsive Disassembly of Polymersomes of SequenceSpecific Boroxole-Containing Block Copolymers under Physiologically Relevant Conditions. ACS Macro Lett. 2012, 1 (10), 1194-1198.

27. Greenspan, L., Humidity fixed points of binary saturated aqueous solutions. J. Res. Natl. Bur. Stand. Sect. A Phys. Chem. 1977, 81 A (1), 89-96.

28. Dreyer, D. R.; Jia, H.-P.; Bielawski, C. W., Graphene Oxide: A Convenient Carbocatalyst for Facilitating Oxidation and Hydration Reactions Angew. Chem., Int. Ed. 2010, 49 (38), 68136816.

29. Dreyer, D. R.; Park, S.; Bielawski, C. W.; Ruoff, R. S., The Chemistry of Graphene Oxide. Chem. Soc. Rev. 2010, 39 (1), 228240.

30. Zhang, X.; Ciesielski, A.; Richard, F.; Chen, P.; Prasetyanto, E. A.; De Cola, L.; Samorì, P., Modular GrapheneBased 3D Covalent Networks: Functional Architectures for Energy Applications. Small 2016, 12 (8), 1044-1052.

31. Lin, Y.; Jin, J.; Song, M., Preparation and Characterisation of Covalent Polymer Functionalized Graphene Oxide. J. Mater. Chem. 2011, 21 (10), 3455-3461.

32. Kuila, T.; Bose, S.; Mishra, A. K.; Khanra, P.; Kim, N. H.; Lee, J. H., Chemical Functionalization of Graphene and its Applications. Prog. Mater. Sci. 2012, 57 (7), 1061-1105.

33. Dreyer, D. R.; Todd, A. D.; Bielawski, C. W., Harnessing the Chemistry of Graphene Oxide. Chem. Soc. Rev. 2014, 43 (15), 5288-5301.

34. Compton, O. C.; Dikin, D. A.; Putz, K. W.; Brinson, L. C.; Nguyen, S. T., Electrically Conductive "Alkylated" Graphene Paper via Chemical Reduction of Amine-Functionalized Graphene Oxide Paper. Adv. Mater. 2010, 22 (8), 892-896.

35. Wang, G.; Shen, X.; Wang, B.; Yao, J.; Park, J., Synthesis and Characterisation of Hydrophilic and Organophilic Graphene Nanosheets. Carbon 2009, 47 (5), 1359-1364.
36. Zhao, Y.; Ding, H.; Zhong, Q., Preparation and Characterization of Aminated Graphite Oxide for CO2 Capture. Appl. Surf. Sci. 2012, 258 (10), 4301-4307.

37. Li, W.; Tang, X.-Z.; Zhang, H.-B.; Jiang, Z.-G.; Yu, Z.-Z.; Du, X.-S.; Mai, Y.-W., Simultaneous Surface Functionalization and Reduction of Graphene Oxide with Octadecylamine for Electrically Conductive Polystyrene Composites. Carbon 2011, 49 (14), 47244730 .

38. Slobodian, O. M.; Lytvyn, P. M.; Nikolenko, A. S.; Naseka, V. M.; Khyzhun, O. Y.; Vasin, A. V.; Sevostianov, S. V.; Nazarov, A. N., Low-Temperature Reduction of Graphene Oxide: Electrical Conductance and Scanning Kelvin Probe Force Microscopy. Nanoscale Res. Lett. 2018, 13 (139), 1-11.

39. Tegou, E.; Pseiropoulos, G.; Filippidou, M. K.; Chatzandroulis, S., Low-Temperature Thermal Reduction of Graphene Oxide Films in Ambient Atmosphere: Infra-Red Spectroscopic Studies and Gas Sensing Applications. Microelectron. Eng. 2016, 159, 146-150.

40. Moldoveanu, S. C., Chapter 8 - Pyrolysis of Amines and Imines. In Pyrolysis of Organic Molecules (Second Edition), Moldoveanu, S. C., Ed. Elsevier: 2019; pp 327-347.

41. Patel, M.; Feng, W.; Savaram, K.; Khoshi, M. R.; Huang, R.; Sun, J.; Rabie, E.; Flach, C.; Mendelsohn, R.; Garfunkel, E.; He, H., Microwave Enabled One-Pot, One-Step Fabrication and Nitrogen Doping of Holey Graphene Oxide for Catalytic Applications. Small 2015, 11 (27), 3358-3368.

42. Wang, Y.; Liao, X.; Luo, Y.; Yang, Q.; Li, G., Influence of Surface-functionalized Graphene Oxide on the Cell Morphology of Poly(methyl methacrylate) Composite. J. Mater. Sci. Technol. 2015, 31 (5), 463-466.

43. Acik, M.; Lee, G.; Mattevi, C.; Pirkle, A.; Wallace, R. M.; Chhowalla, M.; Cho, K.; Chabal, Y., The Role of Oxygen during Thermal Reduction of Graphene Oxide Studied by Infrared Absorption Spectroscopy. J. Phys. Chem. C 2011, 115 (40), 1976119781.

44. Chen, Y.; Zhang, X.; Zhang, D.; Yu, P.; Ma, Y., High Performance Supercapacitors Based on Reduced Graphene Oxide in Aqueous and Ionic Liquid Electrolytes. Carbon 2011, 49 (2), 573-580.

45. Pei, S.; Cheng, H.-M., The Reduction of Graphene Oxide. Carbon 2012, 50 (9), 3210-3228.

46. Ferrari, A. C., Raman Spectroscopy of Graphene and Graphite: Disorder, Electron-Phonon Coupling, Doping and Nonadiabatic Effects. Solid State Commun. 2007, 143 (1), 47-57.

47. Muchharla, B.; Narayanan, T. N.; Balakrishnan, K.; Ajayan, P. M.; Talapatra, S., Temperature Dependent Electrical Transport of Disordered Reduced Graphene Oxide. 2d Materials 2014, 1 (1).

48. Zhang, D.; Tong, J.; Xia, B., Humidity-Sensing Properties of Chemically Reduced Graphene Oxide/Polymer Nanocomposite Film Sensor Based on Layer-by-Layer Nano Self-Assembly. Sens. Actuators, B 2014, 197, 66-72. 